INPLASY

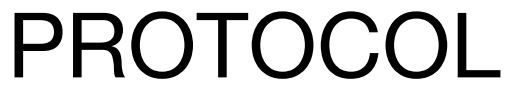

To cite: Xu et al. Efficacy and safety of Jiangzhitongmai capsules for hyperlipidaemia: protocol for a meta-analysis of randomized controlled trials. Inplasy protocol 202110106. doi:

10.37766/inplasy2021.1.0106

Received: 28 January 2021

Published: 28 January 2021

Corresponding author:

Guiqin Xu

26268905@qq.com

Author Affiliation:

The First Affiliated Hospital of Anhui University of Traditional Chinese Medicine

Support: HAVE.

Review Stage at time of this submission: The review has not yet started.

Conflicts of interest:

None.

\section{Efficacy and safety of Jiangzhitongmai capsules for hyperlipidaemia: protocol for a meta-analysis of randomized controlled trials}

$$
\text { Xu, GQ1; Dai, XI2; Hang, C3; Qi, HY4. }
$$

Review question / Objective: Is Jiangzhi tongmai capsules effective and safe in the treatment of hyperlipidaemial? Condition being studied: Participants with primary dyslipidemia.

Information sources: A literature search will be performed in 3 Chinese(China National Knowledge Infrastructure, Wanfang, and VIP) and 4 English (PubMed, Embase, Cochrane Library, Web of science) electronic databases from their inception to Jan 282021.

INPLASY registration number: This protocol was registered with the International Platform of Registered Systematic Review and Meta-Analysis Protocols (INPLASY) on 28 January 2021 and was last updated on 28 January 2021 (registration number INPLASY202110106).

\section{INTRODUCTION}

Review question / Objective: Is Jiangzhi tongmai capsules effective and safe in the treatment of hyperlipidaemial?

Rationale: Hyperlipidemia refers to hypercholesterolemia, hypertriglyceridemia and mixedhyperlipidemia, and is a common biochemical disorder with significantly risk of coronary heart disease. Hyperlipidemia can lead to a serious effect on health. Statins are currently the preferred agent for the treatment of hypercholesterolemia. But there are mangt side effects of statins, such as memory loss, impairment of liver/ kidney function, new onset diabetes and muscle symptoms. And traditional Chinese 
medicine is widely used for hyperlipidemia in China. Jiangzhi tongmai capsule, a kind of Chinese patent medicine, has been widely used to treat hyperlipidemia. However, the effect and safety of Jiangzhi tongmai capsule for treating hyperlipidemia has not been systematically evaluated.

Condition being studied: Participants with primary dyslipidemia.

\section{METHODS}

Search strategy: The search strategy consisted with the following terms: "Jiangzhi tongmai capsule" [MeSH Terms] AND " hyperlipidaemia" [MeSH Terms] AND“Randomized Controlled Trial” [ptyp].

Participant or population: Patients with hyperlipidemia publicly diagnosed.

Intervention: Jiangzhi tongmai capsule or Jiangzhi tongmai capsule combined with the routine treatment used in western medicine.

Comparator: The control group should be treated with the routine treatment used in western medicine or western medicine combined with Chinese medicine.

Study designs to be included: The randomized controlled trials will be contained.

Eligibility criteria: Type of studies:The randomized controlled trials will be contained .Jiangzhi tongmai capsule for patients OR Jiangzhi tongmai capsule with western medicine for patients.without the limitations of age,sex or ethnicity.

Information sources: A literature search will be performed in 3 Chinese(China National Knowledge Infrastructure, Wanfang, and VIP) and 4 English( PubMed, Embase, Cochrane Library, Web of science) electronic databases from their inception to Jan 282021.
Main outcome(s): Primary outcomes are serum lipid levels, including TC, TG, LDL-C, and HDL-C The secondary outcomes will be include Clinical efficacy, adverse events.

Data management: Data from included studies will be extracted following structured forms with the relevant information (e.g.,author's name, publication year, study design, sample size, characteristics of the patients, type of intervention, treatment course, outcomes, adverse events).

Quality assessment / Risk of bias analysis: Two reviewers will use the Cochrane Collaboration's tool to evaluate the risk of bias of included RCTs. The tool includes six aspects such as selection bias(random sequence generation, allocation concealment), performance bias(blinding of participants and researchers), detection bias(blinding of outcome assessment), attrition bias (incomplete outcome data),reporting bias ( selective reporting) and other bias to assess quality. Other more,The corresponding author will be contacted to examine issues if necessary. The included studies will be assessed either as low, high, or unclear risk.

Strategy of data synthesis: RevMan 5.3.5 software from the Cochrane Collaboration will be used to synthesize and analyze the included data. The continuous data will be analyzed by using mean difference with 95\% Confidence interval(Cl), while the analysis of dichotomous data will be analyzed by adopting relative risk ratios with $95 \% \mathrm{Cl}$. The heterogeneity will be assessed based on the results of standard $X 2$ test, if 12 value is more than $50 \%$ indicating high heterogeneous, the random-effect model will be used to pool the data. Otherwise, a fixed-effect model will be applied for data synthesis when 12 value is less than $\mathbf{5 0 \%}$.

Subgroup analysis: Subgroup analysis will be performed to assess possible biasing factors of meta results following the factors of age, sex. 
Sensitivity analysis: Sensitivity analyses will be conducted at factors may be strongly influence the results. For instance, whether the results are different when excluding the low-quality articles.

Country(ies) involved: The systematic review is being carried out in China.

Keywords: Jiangzhitongmai capsule, hyperlipidaemia, systematic review, meta-analysis, randomized controlled trials.

Contributions of each author:

Author 1 - Guiqin Xu.

Author 2 - Xueli Dai.

Author 3 - Cheng Hang.

Author 4 - Haiyan Qi. 